\title{
Incapacidades laborales: problemas en la reinserción al trabajo
}

\author{
Work-related disabilities and workers' \\ difficulties in rejoining the labor market
}

Rosana F. Sampaio 1 Albert Navarro $i$ G. 2 Miguel Martín M. ${ }^{2}$

\footnotetext{
1 Departamento de Fisioterapia, EEF, Universidade Federal de Minas Gerais, Unidade Administrativa II, 2o andar, Av. Antônio Carlos 6627, Pampulha, Belo Horizonte, MG, 31270-901, Brasil.

2 Laboratorio de Estadística y Epidemiología, Facultad de Medicina, Universidad Autónoma de Barcelona, Espanha.
}

\begin{abstract}
This cross-sectional study was conducted on permanently disabled workers assisted by the Center for Occupational Rehabilitation (CRP) in Belo Horizonte, Minas Gerais, Brazil, to evaluate factors associated with their chances of readmission into the labor market. A logistic model was used to investigate workers' acceptance into the professional rehabilitation program and their return into the market. The most decisive factors for their acceptance were age, presence of repetitive strain injury (RSI), and educational level. The most favorable situation was that of workers with RSI under 40 years of age and with average schooling (75.6\%). Results showed that young male workers with high educational levels were the ones with the best chances of rejoining the market (90.9\%). A controversial issue relates to workers' acceptance and program results. In order to be accepted, the worker had to display a given age and educational profile. Workers with RSI had this profile, but once accepted they were the ones with the greatest difficulty in rejoining the work force. In addition, discrimination against female workers was observed, despite their characteristics being similar to those of males.
\end{abstract}

Key words Occupational Accidents; Occupational Health; Supported Employment

Resumen En este estudio transversal se evalúan los factores asociados con la probabilidad de reincorporación al trabajo de los trabajadores incapacitados permanentes para la profesión habitual por accidente de trabajo. La muestra la forman los casos atendidos por el Centro de Rehabilitación Profesional (CRP) de Belo Horizonte, Brasil, en 1995. Se analiza el hecho del trabajador ser aceptado o no en el programa, así como la posterior reinserción laboral mediante modelos logit. Respecto al primer, los factores más influyentes fueron la edad, la presencia de Lesiones por Esfuerzo Repetitivo (LER) y el nivel de estudios. Analizado el resultado del programa, se observa que los hombres jóvenes de alta escolarización son los que tienen mayor probabilidad de reinserción laboral (90,9\%). Existe una incongruencia respecto a la admisión de los trabajadores y el resultado del programa: para su admisión, el trabajador debe cumplir un perfil exigido por el mercado en lo que se refiere a la edad y al nivel de estudios; los portadores de LER cumplen estos criterios y, sin embargo, son los que con mayor dificultad se reincorporan al trabajo. Se observa, así mismo, una discriminación laboral clara hacia las mujeres, a pesar del hecho que las mismas presentan iguales condiciones de edad o de nivel educativo que los hombres. Palabras clave Accidente de Trabajo; Salud Laboral; Readaptación al Trabajo 


\section{Introducción}

En Brasil, el accidente de trabajo (AT) legalmente definido puede ser: típico o tipo, cuando ocurre durante la jornada laboral; de trayecto, si ocurre entre la casa y el trabajo, y finalmente la enfermedad profesional o del trabajo (Lei № 8.213 de 24/07/91) (MPAS, 1995b).

El término incapacidad, objeto central de este trabajo, es definido de distintas maneras en la literatura médica. Capacidad, del latín $c a$ pacitas-tatis, es la aptitud o suficiencia para hacer efectiva algunas cosas y capacidad laboral puede ser entendida como la aptitud para desempeñar de forma eficiente una determinada tarea (Basile \& Gonzaléz, 1983). La definición de las incapacidades laborales tiene en cuenta el grado de compromiso del individuo y el criterio cronológico (temporal o permanente) (Boden, 1995). Bajo un marco legal, las incapacidades permanentes son definidas como aquellas que impiden una actividad laboral habitual en forma definitiva, o sea para el resto de la vida del trabajador (Basile \& Gonzaléz, 1983).

Esta situación se produce cuando el Instituto Nacional de Seguro Social (INSS), en el caso de Brasil, reconoce que las secuelas, de cualquier naturaleza, que afectan el trabajador que sufrió un AT son irreversibles. Estos casos, tras la evaluación por un perito médico, son dirigidos hacia la rehabilitación profesional mediante estos programas desarrollados en Centros de Rehabilitación Profesional (CRP) y la concesión de prestaciones (benefícios) a los trabajadores asegurados (MPAS, 1995a).

Los técnicos que actuan en el programa de rehabilitación profesional del INSS tienen la función de evaluar y definir la capacidad laboral residual del trabajador, orientar la nueva ocupación y procurar información acerca de la reinserción del accidentado en el mercado de trabajo.

Es importante tener claro que, pese todas las atribuciones y competencias del CRP, éste no dispone de ningún instrumento legal para garantizar el retorno del trabajador rehabilitado a la empresa de origen y tampoco desempeña la función de encontrar un puesto de trabajo para los accidentados. Es decir, la responsabilidad del Centro acaba en el alta del trabajador considerado por ellos rehabilitado. El laudo final ofrecido por el CRP tampoco es decisivo, ya que es el perito médico quién puede determinar cómo y cuando finalizará la relación del trabajador incapacitado con el INSS.

En este contexto, el objetivo de este trabajo es caracterizar a los trabajadores incapacitados permanentes para la profesión habitual por AT y evaluar los factores asociados con la probabilidad de reincorporación al mundo laboral.

La decisión de utilizar datos del INSS (CRP) fue debida a la falta de información acerca de la incapacidad permanente por AT en otras instancias. Esta fuente de información tiene algunas limitaciones que merecen ser discutidas. Desde el punto de vista poblacional, es importante destacar que la información se restringe a los trabajadores que pertenecen a la Seguridad Social, es decir, desconocemos lo que ocurre con el resto de los trabajadores incapacitados permanentes no inscritos en el INSS.

Otro aspecto a tener en cuenta es el de la dificultad en definir denominadores para el cálculo de grupos de riesgos debido a la extensa cobertura del CRP de Belo Horizonte. Por último no podemos olvidarnos de que los informes del INSS tienen un carácter indemnizador y, por lo tanto, la información que vamos a utilizar en este análisis responde a esta lógica.

En modo alguno estas consideraciones disminuyen la importancia del presente estudio, solamente delimitan dónde estamos y hasta dónde podremos avanzar. Considerando que la cantidad de trabajos producidas en Brasil hasta el momento no es proporcional a la dimensión del problema (Cohn et al., 1985; Struffaldi, 1987; Struffaldi, 1994; MPAS, 1995a), este trabajo nos posibilitará construir los contornos iniciales del diagnóstico de la situación de las incapacidades permanentes para la profesión habitual por AT en Belo Horizonte.

\section{Material y método}

Es un estudio transversal (1995) de los trabajadores incapacitados permanentes para la profesión habitual, sometidos a programa en el CRP/INSS de Belo Horizonte, Minas Gerais, Brasil. Se utilizaron datos secundarios, extraídos del historial médico, abierto a partir del registro del trabajador en el CRP.

\section{Variables analizadas}

\section{a) Datos socio-demográficos:}

1) Sexo.

2) Edad recodificada según la População Economicamente Ativa (PEA), inicialmente en 5 categorías: hasta 19 años; 20 hasta 29 años; 30 hasta 39 años; 40 hasta 49 años y más de 50 años.

3) Estado civil en 4 categorías: soltero, casado, separado o divorciado y viudo.

4) Escolaridad: inicialmente recodificada en 8 categorías: analfabetos (se consideran 
analfabetos a los trabajadores que no saben leer, en algunos casos son capaces solamente de firmar su nombre), semianalfabetos (trabajadores que, aunque no presentan escolaridad formal, son capaces de realizar cálculos matemáticos básicos (sumas y restas) y tienen poca capacidad de lectura), primer grado completo, primer grado incompleto, segundo grado completo, segundo grado incompleto, curso universitario completo y curso universitario incompleto.

b) Motivo del ingreso en el programa de rehabilitación profesional: variable recodificada conforme los criterios del INSS en 3 categorias: accidente típico, accidente de trayecto y enfermedad profesional.

c) Información sobre el proceso de trabajo:

1) Ocupación: recodificada según la Classificação Brasileira de Ocupação (CBO) (MT, 1994), inicialmente a tres dígitos y posteriormente a uno sólo.

2) Rama de actividad económica categorizada según la Classificação Nacional de Atividades Econômicas (CNAE) (Manuais de Legislação Atlas, 1996) a dos dígitos.

3) Sector económico: agrupado en 4 categorías a partir de la CNAE: agricultura y pecuaria, industria, construcción y servicios.

d) Antigüedad del trabajador en el puesto de trabajo hasta la fecha del accidente: el tiempo de permanencia en el trabajo hasta la fecha del accidente fue informatizado en días. Posteriormente, fue agrupado en 6 categorías: 0 hasta 6 meses, 6 meses hasta 2 años, 2 hasta 4 años, 4 hasta 9 años, 9 hasta 14 años y más de14 años.

e) Situación del trabajador en el mercado de trabajo: se considera la situación del trabajador en el mercado de trabajo declarada en el momento del registro en el programa de rehabilitación profesional: asalariado, autónomo, jubilado y desempleado.

f) Diagnóstico: el diagnóstico registrado con cinco dígitos según la Classificação Internacional de Doenças (CID, 1975), fue posteriormente recodificado a tres y a un solo dígito.

g) Aceptación en el programa: aceptado y no aceptado.

h) Resultado del programa: vuelve o no vuelve al trabajo.

El análisis descriptivo de los datos, así como la recodificación de variables, se ha llevado a cabo con el programa SPSS 6.0. En el análisis bivariado de las dependencias entre las variables categóricas se ha utilizado el estadístico del chi-cuadrado y, en caso de no cumplirse las condiciones de aplicabilidad, las hipótesis de independencia se han contrastado mediante el test exacto de Fischer con el programa StatXact
2.11. En los contrastes de medias, se ha utilizado el test de la t de Student y el de Mann-Withney en el caso de contraste entre medianas. En todos los casos se ha considerado estadísticamente significativo la obtención de un p-valor inferior a 0,05 .

El análisis multivariado se ha efectuado mediante el ajuste de modelos logit a partir del log-lineal correspondiente (Fischer, 1993; Sampaio, 1995), con el fin de determinar los factores que se asocian a la no aceptación del trabajador en el programa de rehabilitación profesional, así como al resultado final del programa.

Para ello, se han considerado las variables que muestran una asociación con las respuestas en el análisis bivariado previo, así como los posibles factores de confusión de dichas asociaciones. Por lo tanto, se han ajustado dos modelos, uno para cada variable respuesta:

1) Aceptación en el programa de rehabilitación profesional; la variable respuesta, ACP, posee dos posibles categorias: ACP (1), correspondiente a la no aceptación en el programa y ACP (2) para los trabajadores aceptados.

2) Resultado del programa en los trabajadores aceptados: la variable respuesta, VUE, tiene dos categorias, VUE (1) para los trabajadores aceptados en el programa de rehabilitación profesional que no se reincorporan al mercado laboral, y VUE (2), para los que logran volver al mundo del trabajo.

Debido a la importancia actual del tema de las enfermedades de trauma repetitivos cuanto a las patologías de origen laboral y a la proporción de casos encontrados en este estudio, se dividió el análisis en dos grupos: trabajadores con enfermedad de trauma repetitivo y el resto. En ambos modelos, los factores considerados son: portador de lesión por esfuerzo repetitivo, LER, en dos categorias: presencia o ausencia.

La variable sexo, SEX, en dos categorias: hombre o mujer.

La edad se considera en dos categorias: edad igual o superior a los 40 años o menor de 40 años.

La escolaridad, ESC, se categoriza en tres niveles: la primera se asigna a los trabajadores analfabetos o semianalfabetos, la segunda a los que poseen escolaridad comprendida entre el primer grado completo o incompleto y segundo grado incompleto y, por ultimo, la tercera categoria en el caso de haber cursado el segundo grado completo y curso superior completo o incompleto.

Con el fin de valorar el efecto de estos factores, se han seleccionado unas situaciones observacionales de referencia: aceptación en el programa, la odds ACP(2)/ACP(1) de referencia 
es la de los trabajadores varones sin LER, mayores de 40 años cumplidos y del primer nivel de escolaridad (analfabeto/semianalfabeto).

En el caso de la odds VUE(2)/VUE(1), la situación de referencia es distinta y es la conformada por trabajadoras portadoras de LER, también de edad de 40 años o más y del primer nivel de escolaridad (analfabeto/semianalfabeto).

Para este análisis, se ha utilizado el programa GLIM, y los resultados se expresan en términos de proporción de trabajadores aceptados o que vuelven al trabajo, según el modelo considerado. Dichas proporciones se han obtenido mediante la transformación inversa de los logits ajustados de dichos modelos.

\section{Resultados}

Fueron revisados 920 historiales médicos, entre los que predominaron los casos producidos por las enfermedades profesionales $(67 \%)$, seguidas por los accidentes típicos $(31 \%)$ y por los accidentes de trayecto ( $2 \%$ ). Un $58 \%$ de los casos revisados eran hombres y el $42 \%$, mujeres. La media de edad encontrada fue de 34 años (DE: 8,78), similar entre hombres y mujeres. Con relación al estado civil, un $63 \%$ de los trabajadores estaba casado, $31 \%$ soltero, $5 \%$ separado y el $1 \%$ era viudo. Un $50 \%$ de los trabajadores tenía el nivel educativo correspondiente al primer grado completo o incompleto y la mayor parte de ellos eran asalariados (90\%), es decir, se encuentraban formalmente insertados en el mercado de trabajo.

Las cuatro ocupaciones (CBO a tres dígitos) más frecuentes fueron: auxiliares de escritorio $(9,4 \%)$, seguidos por auxiliares contables/empleados de banco (caja) $(8,5 \%)$, trabajadores de servicios de manutención y limpieza $(7,7 \%)$ y, finalmente, operadores de máquinas de procesamiento de datos $(6,4 \%)$. En cuanto a la rama de actividad (CNAE a dos dígitos), destacan las instituciones financieras, seguidas por la construcción, metalurgía básica y servicios prestados a las empresas. En la rama de servicios prestados a las empresas, se encuentran los trabajadores temporales de la limpieza y vigilancia.

En relación a la antigüedad en el trabajo, el patrón es diferente para el caso de accidentes y de enfermedades profesionales. Entre los primeros, casi la mitad llevaban menos de 6 meses trabajando; por el contrario, en las enfermedades profesionales un $26 \%$ llevaba de 4 hasta 9 años, seguido de una cuarta parte que tenía entre 6 meses y 2 años de antigüedad en el trabajo.

Más de la mitad de los casos (53\%) aquí analizados fueron diagnosticados como LER
(CID a tres dígitos), seguidos por amputaciones traumáticas de los dedos de la mano $(3,4 \%)$, efectos tardios de lesiones traumáticas osteomusculares $(3,3 \%)$, dermatitis de contato $(3,2 \%)$ y pneumoconiosis debido a sílice $(3,1 \%)$.

De los 920 casos registrados y descritos anteriormente, un 35\% ( $n=322)$ no fueron aceptados para programa, después de evaluados por los técnicos del Centro. Entre los 592 casos evaluados y aceptados para la rehabilitación profesional, un $25 \%$ ( $n=142$ ) fueron considerados sin posibilidad de vuelver al trabajo después de iniciada la rehabilitación profesional.

En la Tabla 1 presentamos el modelo logit para la aceptación en el programa de rehabilitación profesional. Por los resultados, pudemos ver que ser hombre o mujer prácticamente no afecta el hecho de ser aceptado en el programa. La situación óptima de aceptación es la de los trabajadores portadores de LER, menores de 40 años y con el nivel de estudios medio (primer grado completo o incompleto y segundo grado incompleto), para nosotros ESC (2). En esta situación, la probabilidad estimada de aceptación es del 75,6\%.

El resultado del programa para los trabajadores aceptados en el mismo se presenta en la Tabla 2. El diagnóstico, o sea, la presencia o no de LER, no influye en el resultado del programa. La vuelta al trabajo es óptima para aquellos trabajadores varones, menores de 40 años y del más alto nivel de escolarización (segundo grado completo y curso superior completo o incompleto). En esta situación la reincorporación al mundo laboral es del 90,9\%.

\section{Discusión}

El análisis de la información extraída de los historiales médicos nos permitió caracterizar a las personas incapacitadas por accidente de trabajo y orientadas hacia el programa de rehabilitación profesional del INSS. La discusión de los resultados tiene dos desdoblamientos importantes: uno que dice respecto a los factores relacionados con la ocurrencia de la incapacidad permanente para la profesión habitual y el otro que son las consecuencias de la misma en cuanto al retorno del trabajador al mundo laboral. En este estudio, estas consecuencias pueden ser evaluadas por todo el proceso vivido por los trabajadores después del accidente, desde su aceptación o no en el programa de rehabilitacion profesional hasta los resultados del mismo.

La edad de los incapacitados muestra la gravedad de la situación de los accidentes de 
trabajo: los trabajadores se quedan incapacitados con menos de 40 años, es decir, en plena fase productiva y reproductiva. La repercusión social de este hecho es enorme, principalmente cuando el Estado, con su política neoliberal, intenta difundir la idea de que la Seguridad Social atraviesa una crisis económica que motivará cortes en el sistema y, consecuentemente, reducción de los derechos sociales.

No hay una relación de causalidad directa entre escolaridad y accidentes de trabajo. La escolaridad determina la inserción del trabajador en el proceso productivo, y las diferencias entre estos procesos es que resultan en el mayor o menor número de accidentes. Asimismo, algunos autores afirman que los trabajadores con escolaridad más elevada se encuentran mejor informados cuanto a sus derechos y, por lo tanto, se muestran más reacios a aceptar un trabajo en condiciones arriesgadas (Cohn et al., 1985). Si, por un lado, la literatura apunta para la insuficiencia de nexos de causalidad entre accidente y escolaridad, ésta desempeña un papel primordial en cuanto a la rehabilitación profesional del trabajador, no sólo en este Centro como también en otros países (Cornes et al., 1986; MacKenzie et al., 1987; Aitken \& Cornes, 1990).

Debe tenerse en cuenta que la información disponible en este estudio, sobre la situación del trabajador en relación al mercado laboral, no refleja la realidad del mercado de Belo Horizonte. El elevado número de asalariados es consecuencia de la exigencia legal que coloca mayoritariamente este tipo de trabajador como contribuyente del INSS y, por lo tanto, quién tiene prioritariamente el derecho a estar en el CRP. De todos modos, el vinculo del trabajador con una empresa debería facilitar su reinserción en una función que sea compatible con sus capacidades después del accidente, pero eso no siempre suele ocurrir, según la opinión de los técnicos del Centro (CRP-BH, 1996) y los resultados de este estudio. Con mucha frecuencia, los empleadores no se sienten comprometidos con el accidentado del trabajo o portador de enfermedad profesional.

Los resultados aquí encontrados respecto a la ocupación y rama de actividad económica evidencian que son las condiciones de trabajo diferenciadas, producción y no producción, las que determinan las repercusiones del trabajo sobre la salud de los trabajadores. El predominio de accidentes en el sector industrial y de enfermedades profesionales en servicios viene a reafirmar esta constatación.

El hecho de que $44 \%$ de los accidentes ocurrieron entre trabajadores que tenían menos de
Tabla 1

Probabilidad de ser aceptado en el programa según el modelo logístico.

\begin{tabular}{lcccc}
\hline Situación & Odds & OR & Probabilidad & $\begin{array}{l}\text { Razón de } \\
\text { probabilidad }\end{array}$ \\
\hline Basal & 0,5872 & & 0,370 & \\
Portador de LER & 0,9855 & 1,678 & 0,496 & 1,342 \\
Mujer & 0,5919 & 1,008 & 0,372 & 1,005 \\
$<40$ años & 0,9996 & 1,702 & 0,499 & 1,351 \\
2ndo nivel escolar & 1,0863 & 1,850 & 0,527 & 1,407 \\
3er nivel escolar & 0,9246 & 1,575 & 0,480 & 1,299 \\
\hline
\end{tabular}

* 1 er nivel escolar: analfabeto/semianalfabeto.

2ndo nivel escolar: primer grado completo o incompleto/segundo grado incompleto. 3er nivel escolar: segundo grado completo/curso superior completo o incompleto.

Tabla 2

Probabilidad de volver al trabajo según el modelo logístico.

\begin{tabular}{lcccc}
\hline Situación & Odds & OR & Probabilidad & $\begin{array}{l}\text { Razón de } \\
\text { probabilidad }\end{array}$ \\
\hline Basal & 0,0624 & & 0,059 & \\
No LER & 0,1107 & 1,773 & 0,099 & 1,696 \\
Hombre & 0,1492 & 2,390 & 0,130 & 2,209 \\
$<40$ años & 0,1606 & 2,573 & 0,138 & 2,355 \\
2ndo nivel escolar & 0,5148 & 8,248 & 0,340 & 5,785 \\
3er nivel escolar & 0,9231 & 14,791 & 0,480 & 8,171 \\
\end{tabular}

* 1 er nivel escolar: analfabeto/semianalfabeto.

2ndo nivel escolar: primer grado completo o incompleto/segundo grado incompleto. 3er nivel escolar: segundo grado completo/curso superior completo o incompleto.

seis meses de antigüedad en el trabajo no nos sirve para medir la asociación entre experiencia y accidentalidad. Esta variable representa tan sólo el tiempo que llevaba trabajando hasta la fecha del accidente. De todos modos, es alto el número de trabajadores accidentados antes de completar seis meses trabajando y es importante el hallazgo respecto a la disminución de los mismos en la medida en que aumenta el tiempo de permanencia en el trabajo. Por otro lado, las enfermedades profesionales, que en el pasado presentaban un tiempo de latencia mayor, hoy ya se manifiestan antes de los dos años de exposición.

La rehabilitación profesional, de modo general, exige determinadas características del paciente para alcanzar sus objetivos. En otras palabras, el rehabilitando tiene que ser capaz física y intelectualmente de ser reeducado. El CRP de Belo Horizonte, según los resultados de este estudio, tiene un perfil ya definido de los 
trabajadores en los cuales se deberá invertir esfuerzos en el sentido de intentar reeducarles en nueva ocupación. A partir de la definición de este perfil, queda claro que la rehabilitación profesional del INSS no es un derecho del accidentado del trabajo, sino que existe un proceso de seleción riguroso para el ingreso del trabajador en el Centro. Esta selección no puede ser entendida como consecuencia del deterioro físico del trabajador después del accidente. Un trabajador seriamente lesionado en el trabajo será considerado inválido para el ejercicio profesional e inmediatamente será jubilado. Los casos analizados en este estudio son de trabajadores portadores de una incapacidad permanente que puede ser considerada parcial. Esta incapacidad al principio lo limita para la actividad que ejercía anteriormente pero no para el trabajo en general, es decir, otros son los factores que llevan al rechazo del trabajador por parte del CRP de Belo Horizonte.

El análisis multivariado nos permitió detectar un sesgo muy importante en las normas adoptadas por este Centro respecto a la admisión de los trabajadores y el resultado del programa. Para ser aceptado, el trabajador debe tener el perfil exigido por el mercado, es decir, básicamente la escolaridad y la edad definen la entrada del trabajador. Por los resultados presentados son los portadores de LER quién tienen estas características. Sin embargo, después de admitidos estos trabajadores son rehabilitados en menor número.

\section{Agradecimientos}

Este estudio fue financiado por el Conselho de Desenvolvimento Científico e Tecnológico (CNPq)/Brasil.
Parece ser que tales criterios repiten la discriminación del mercado laboral respecto a las mujeres (Kon, 1994; Pires, 1994; Maquieira \& Vara, 1997), considerando que las mismas presentan la edad y la escolaridad exigida pero son consideradas aptas para volver al trabajo en menor proporción que los hombres. En certo modo, la filosofia del programa de rehabilitación del INSS/Belo Horizonte refleja la segmentación genérica del mercado de trabajo y el papel simbólico y ideológico que las mujeres ocupan en el mismo. Seguramente, este hecho repercute en la efectividad de la rehabilitación, pues, de este modo, es muy reducido el número de trabajadores que se benefician del programa.

En cualquier caso, si no se crean instrumentos legales que obliguen a las empresas a que se comprometan con la reinserción de los lesionados en el trabajo, principalmente de las mujeres, la rehabilitación profesional ofrecida por el Estado es solamente un paliativo para el problema. Con el aparecimiento de la LER, parece ser que el criterio escolaridad y edad no cumplen sus funciones de selecionar a los trabajadores en que vale la pena invertir en su reeducación profesional.

Los resultados comentados anteriormente nos muestran, por un lado, la urgencia en invertirse esfuerzos en el sentido de conocer no sólo la etiologia de los accidentes, sino también profundizar en las consecuencias de los mismos y, por otro lado, la importancia de adoptar medidas más rigurosas y efectivas para evitar la ocurrencia de los accidentes.

\section{Referências}

AITKEN, R. C. \& CORNES, P., 1990. To work or not work: That is the question. British Journal of Industrial Medicine, 47:436-441.

BASILE, A. A. \& GONZALEZ, O. S., 1983. Las incapacidades múltiples en Medicina Legal del Trabajo. Trabajo y Salud, jun/jul:3-8.

BODEN, L. I., 1995. Workers compensation in the United State: High costs, low benefits. Annuary Review of Public Health, 16:189-218.

CRP-BH (Centro de Reabilitação Profissional de Belo Horizonte), 1996. Relatório Descritivo do Seguimento dos Segurados Desligados do CRP/BH no $2 \underline{o}$ Semestre de 1993. Belo Horizonte: Instituto Nacional de Seguro Social. 
CID (Classificação Internacional de Doenças.), 1975. 9a Revisão, Adaptada para Uso em Processamento de Dados. Porto Alegre: Sagra.

COHN, A.; KARSCH, S. U.; HIRANO, S. \& SATO, K. A., 1985. Acidente de Trabalho: Uma Forma de Violência. São Paulo: Editora Brasiliense.

CORNES, P.; BOCHEL, H. M. \& AITKEN, R. C. B., 1986. Rehabilitation and return to work of employer liability claimants. International Journal of Rehabilitation Research, 9:119-128.

FISCHER, B. G., 1993. A Methodology for the Health Sciencies Categorical Data: Contingency Tables. New York: Wiley-Interscience.

KON, A. A., 1994. A segmentação ocupacional brasileira segundo gênero. In: IX Encontro Nacional de Estudos Populacionais - ABEP, Resumos (Associação Brasileira de Estudos Populacionais, org.), pp. 235-240, Caxambu: ABEP.

MacKENZIE, E. J.; SHAPIRO, B. S.; SMITH, R. T.; SIEDEL, J. H. \& MOODY, M., 1987. Factors influencing return work following hospitalization for traumatic injury. American Journal of Public Health, 77:329-334.

MANUAIS DE LEGISLAÇÃO ATLAS, 1996. Segurança e Medicina do Trabalho. São Paulo: Editora Atlas.

MAQUIEIRA, V. \& VARA, M. J., 1997. Género, Clase y Etnía en los Nuevos Procesos de Globalización. Madrid: Instituto Universitário de Estudios de la Mujer.

MPAS (Ministério da Previdência e Assistência Social), 1995a. Relatório Anual: Reabilitação Profissional. Belo Horizonte: Superintendência Estadual de Minas Gerais, Instituto Nacional de Seguro Social.
MPAS (Ministério da Previdência e Assistência Social), 1995b. Plano de Benefícios da Previdência Social. Lei № 8.2113 de 23/07/1991 e Decreto № 611 de 21/07/1992. 4a edição. Brasília: MPAS.

MT (Ministério do Trabalho), 1994. Classificação Brasileira de Ocupações. Brasília: Secretaria de Políticas de Emprego e Salários.

PIRES, S. L. E., 1994. Sobre o sexo e a idade do emprego assalariado: Notas sobre a divisão sexual do trabalho no Brasil. In: IX Encontro Brasileiro de Estudos Populacionais, Resumos (Associação Brasileira de Estudos Populacionais - ABEP, org.), pp. 409-443, Caxambu: ABEP.

SAMPAIO, R. F., 1995. Análisis de los Accidentes Laborales Ocurridos en Barcelona Ciudad (1988/1993). Tesis de Doctorado, Barcelona: Faculdad de Medicina, Universidade Autónoma de Barcelona.

STRUFFALDI, M. C. B., 1987. Conhecimentos, Opinião e Conduta dos Médicos-Peritos Acidentários, Face à Reabilitação Profissional do INPS do Município de São Paulo. Dissertação de Mestrado, São Paulo: Faculdade de Saúde Pública, Universidade de São Paulo.

STRUFFALDI, M. C. B., 1994. Reabilitação Profissional: Características, Conhecimentos e Opinióes de Trabalhadores Acidentados. Tese de Doutorado, São Paulo: Faculdade de Saúde Pública, Universidade de São Paulo. 